International Journal of Advanced Chemistry, $8(1)(2020) 123-127$
International Journal of Advanced Chemistry
SPC
Website: www.sciencepubco.com/index.php/IJAC
Research paper

\title{
Synthesis and characterization of pullulan derived hydrogel matrix as carrier for slow release of anti-cancer drug
}

\author{
Kabiru Bello ${ }^{1,2}$ * \\ ${ }^{1}$ Department of Post-graduate Studies and Research in Industrial Chemistry, Mangalore University, \\ Mangalagangothri - 574 199, Karnataka State, India \\ ${ }^{2}$ Department of Science Laboratory Technology, Umaru Ali Shinkafi Polytechnic, Sokoto \\ *Corresponding author E-mail:kbliman7@yahoo.com
}

\begin{abstract}
The present study is aimed at designing a pullulan based hydrogel through grafting technique for slow release of fluorouracil drug. To achieve this, pullulan grafted acrylic acid was synthesized and characterized by FTIR, and FESEM analyses. Swelling was done in different $\mathrm{pH}$ solutions with better swelling in $\mathrm{pH} 2$. As such, the drug loading was done in the specified $\mathrm{pH}$ environment. The release profile revealed that more than $90 \%$ of the drug could be released within 5 days. The prepared hydrogel may be considered as stimuli responsive materials for oral drug delivery of anti-cancer drugs.
\end{abstract}

Keywords: Pullulan; Grafting; Fluorouracil; Anti-Cancer.

\section{Introduction}

The focus of present-day research in the area of biomedicine lies in the development of biodegradable, biocompatible and cost effective drug carriers for controlled release of drugs to the targeted sites. The material in need should be able to retain and release the drug to the desired site in human body. The overall essence is to ameliorate problems such as drug bioactivity and side effect [1] [2].

Hydrogels, because of their ability to hold and retain large amount of fluids, could be used for controlled drug delivery. Additionally, hydrogels are unique in their response to different stimuli such as $\mathrm{pH}$, ionic strength and so forth [3]. Introduction of polysaccharides such as cellulose, chitosan and pullulan in the preparation of hydrogels helps in the formation of hydrogel with better biodegradability, biocompatibility and cost reduction [4 - 8].

Pullulan is a straight chained fungal-driven polysaccharide consisting mainly of three glucose unit interconnected together by an $\alpha-1,4-$ glycosidic bond [9]. The hydrophilic groups in pullulan structure endows it with a tremendous water absorption capacity and could therefore be use as drug carrier material for controlled drug delivery and in the field of water purifications [10]

Acrylic acid is a monomer endowed with carboxyl groups attached to carbon atom of the chain. The carboxyl group found in acrylic acid makes it anionic in solution of neutral $\mathrm{pH}$ [11]. Hence, polyacrylic acid polymer is polyelectrolyte polymer having the ability to absorb, retain and swell in water [12] and can therefore be used in diaper industry, cosmetic and drug delivery.

Fluorouracil, a renowned anti-cancer drug, is fluorine containing organic compound which is soluble in water and could therefore be absorbed and retain by the hydrogel for controlled release to the targeted site. Its hydrophilic site could easily form bond with the hydrophilic moiety of the hydrogel which may dissociate upon slight changes in $\mathrm{pH}$ and ionic strength. This aids the release of drug to the targeted area.

In this submission, pullulan grafted acrylic acid hydrogel was prepared through free radical grafting process. The hydrogel was characterized with FT-IR and FESEM for functional groups and morphological determination. The prepared material was examined for its water uptake capacity through batch sorption technique. Drug loading/release analysis was performed on the material with $90 \%$ of the drug being released in 5 days.

\section{Methodology}

\subsection{Materials}

The pullulan and acrylic acid which were used in the preparation of hydrogel were procured from Sigma Aldrich chemicals Mumbai, India. Fluorouracil was freely given by Syngenta pharmaceutical industry, Mangalore, India. All chemicals/reagents used in the conduct of this research were of analytical grade and were used as received. 


\subsection{Grafting process}

Grafting method was used in the preparation of the hydrogel wherein; a known quantity of pullulan was allowed to completely dissolve in water. To this solution certain amount (10\% each of initiator. Crosslinker and monomer) was added and allowed to swirled for $4 \mathrm{~h}$ at a temperature of $50{ }^{\circ} \mathrm{C}$. Afterwards, the hydrogel was cooled under room temperature and washed with acetone and distilled water. This was to allow for dissolution of unreacted species. The hydrogel was dried in oven till constant weight and stored in desiccators for future use.

\subsection{Characterization}

The prepared hydrogel was characterized for the presence of functional group using Fourier Transform Infrared (FT-IR) spectroscopic technique whilst its morphological characterization was done using Field Emission Scanning Electron Microscopy (FESEM).

\subsection{Swelling studies}

The water uptake capacity of the prepared hydrogel was evaluated by transferring a pre-weighed amount of hydrogel in a known quantity of distilled water and allowed to swell over a specified time frame. The hydrogel was taken out and weighed at regular interval. The same procedure was followed in studying the effect of $\mathrm{pH}$ on water uptake capacity of the hydrogel. The water uptake capacity was calculated using Eq. 1.

$$
\mathrm{Q}_{\mathrm{eq}}=\frac{\mathrm{M}_{2}-\mathrm{M}_{1}}{\mathrm{M}_{1}}
$$

Where; $\mathrm{Q}_{\mathrm{eq}}, \mathrm{M}_{1}$ and $\mathrm{M}_{2}$ are the equilibrium swelling, weight of dry and swollen hydrogel respectively.

\subsection{Drug loading/release experiment}

To investigate the fluorouracil release profiles of the samples, the drug-loaded hydrogel $(0.2 \mathrm{~g})$ was immersed in Erlen-Meyer containing $25 \mathrm{~mL}$ buffer solution ( $\mathrm{pH} 1.2$ and 7.4) while shaking in a shaker (100 rpm) at $27{ }^{\circ} \mathrm{C}$. At desired time intervals, $2 \mathrm{~mL}$ of an aliquot of the solution containing drug-loaded hydrogel was withdrawn and replaced with $2 \mathrm{~mL}$ fresh buffer solution. The concentration of fluorouracil was determined by UV-visible spectrophotometry and the released fluorouracil from hydrogels was measured from the calibration graphs $\left(\mathrm{R}^{2}=0.99\right)$. The cumulative release of drug from the samples was calculated.

\section{Results and discussion}

\subsection{Grafting process}

Grafting method was used in the preparation of the hydrogel wherein; a known quantity of pullulan was allowed to completely dissolve in water. To this solution certain amount (10\% each of initiator. Crosslinker and monomer) was added and allowed to swirled for $4 \mathrm{~h}$ at a temperature of $50{ }^{\circ} \mathrm{C}$. It is proposed that mild temperature treatment of the solution generate active sites on the pullulan which allowed for grafting of acrylic acid onto pullulan during what is known as propagation stage. The chain growth is put to halt by the vinyl groups of crosslinker via termination stage. The proposed mechanism for free radical grafting process is presented in Scheme 1.

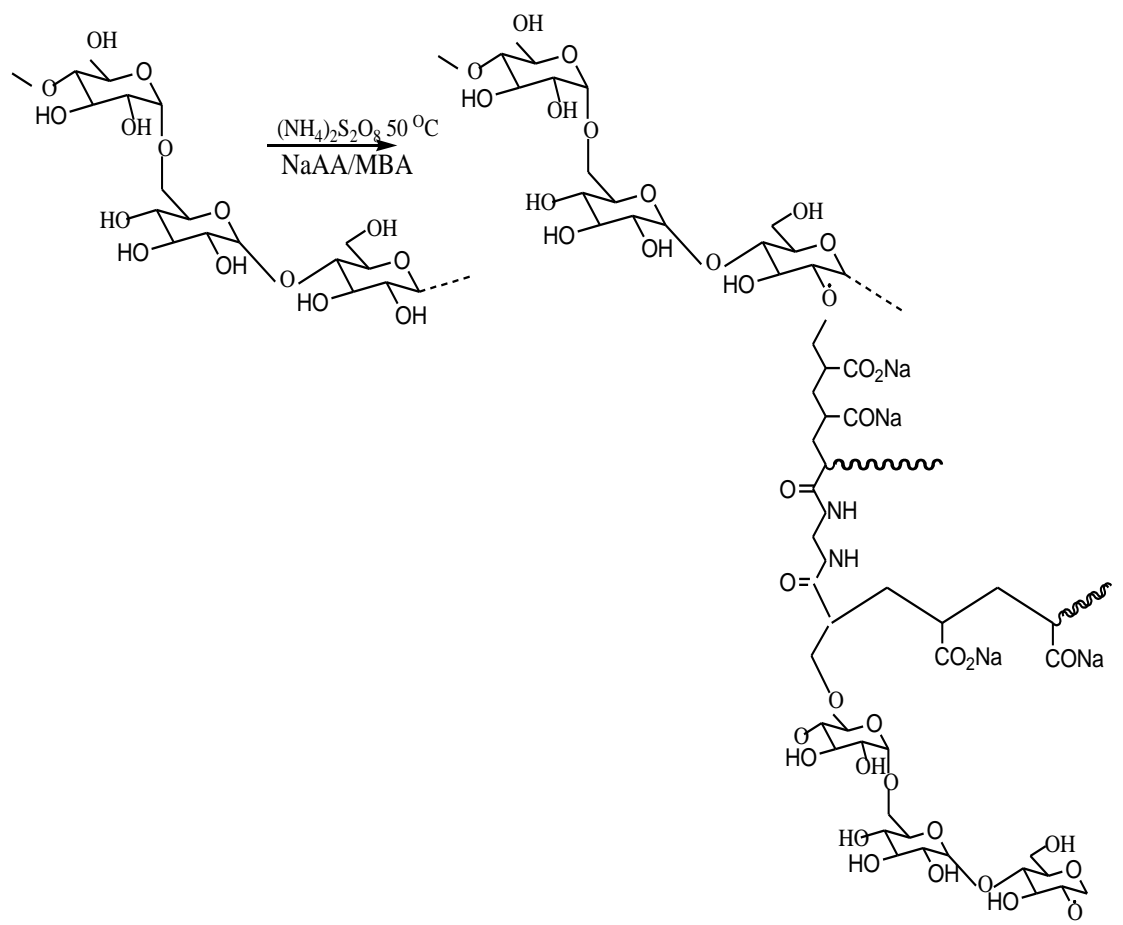

Scheme 1: Proposed Reaction Mechanism for the Preparation of Pullulan Grafted Acrylic Acid. 


\subsection{Fourier-transform infrared spectroscopy}

The identification of functional groups present in the structure of pristine pullulan and the hydrogel was performed using FT-IR spectroscopic analysis using attenuated transmission method and the spectra were transcribed between $4000-400 \mathrm{~cm}^{-1}$ frequency ranges. The spectra were depicted in Fig. 3.1 (a \& b). in addition to the presence of characteristics peaks at 3302, 2916, 1641, 1355, 1171, 1019, 985 and $851 \mathrm{~cm}^{-1}$ which might be assigned to $-\mathrm{OH}$ stretching vibration, $\mathrm{SP}^{3}-\mathrm{C}-\mathrm{H}$ bond, $-\mathrm{O}-\mathrm{C}-\mathrm{O}$ stretching vibration, -C-O-H bending vibration, C-O stretching vibration, 1,6-D-glucosidic bond and D-glucopyranose respectively [13], The FT-IR spectrum of the prepared hydrogel portrayed additional peak at $1725 \mathrm{~cm}^{-1}$ which might be attributed to the carbonyl group absorption found in acrylic acid. This was taken as evidence to confirm the grafting process.
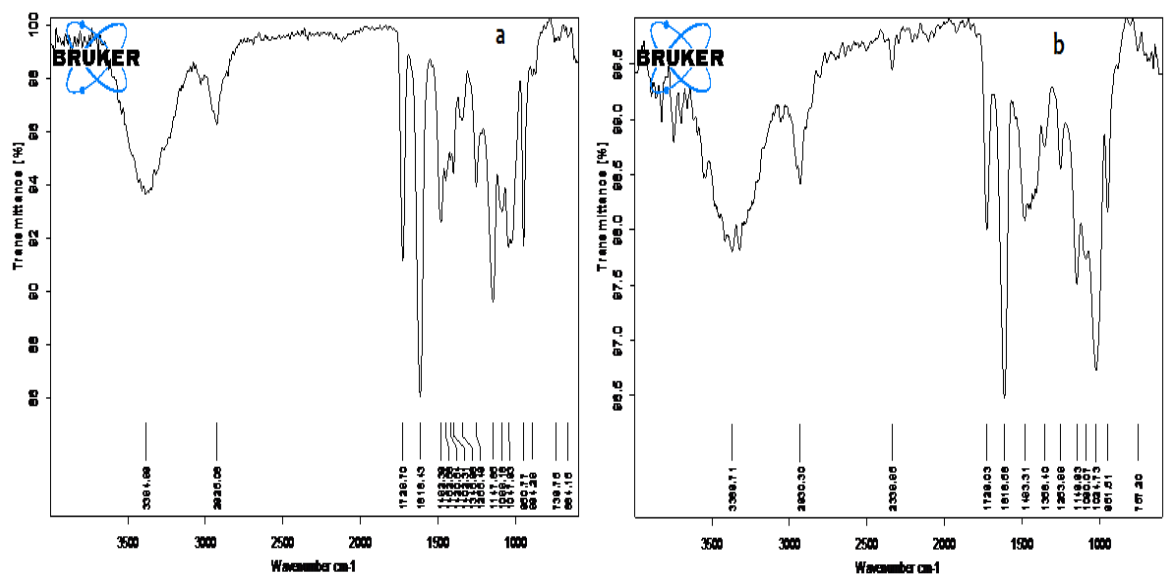

Fig. 3.1: FT-IR Spectrum of (a) Pullulan and (b) Pullulan Grafted Polyacrylic Acid.

\subsection{Field emission scanning electron microscopy (FESEM) analysis}

The surface morphologies of pristine pullulan and the prepared hydrogel are shown in Fig 3.2. These were analyzed using Field Emission Scanning Electron Micrograph. It can be observed that, PL exhibited a smooth surface indicating total dissolution of pullulan in distilled water. The hydrogel displays smooth surface but with agglomerated particles. This agglomerated surface may be due to the grafting of polyacrylic acid on the surface of pullulan. This was used as evidence for successful grafting of acrylic acid onto pullulan backbone.

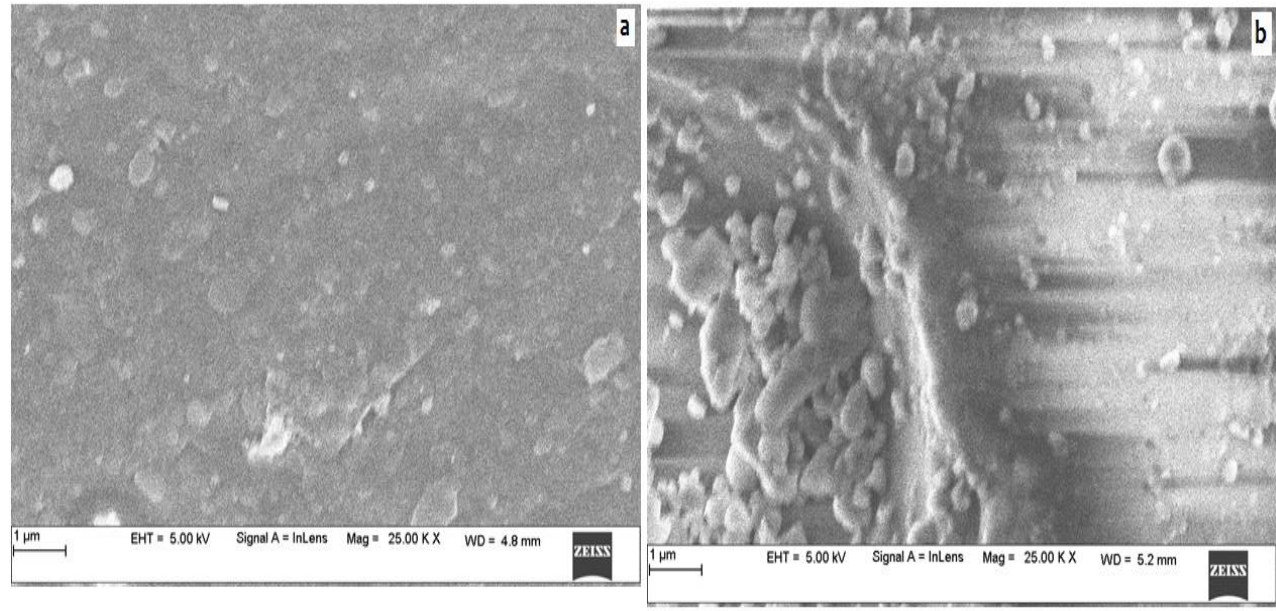

Fig. 3.2: FESEM Images of (a) Pullulan and (b) Pullulan Grafted Polyacrylic Acid Hydrogel.

\subsection{Swelling studies}

The water uptake capacity of the prepared hydrogel was evaluated by transferring a pre-weighed amount of hydrogel in a known quantity of distilled water and allowed to swell over a specified time frame. This was done in order to study the effect of contact time and $\mathrm{pH}$ of solution on swelling behavior of the hydrogel. To study the effect of contact time on swelling, the hydrogel was allowed to swell for a total period of $60 \mathrm{~min}$. maximum swelling was observed at $50 \mathrm{~min}$ with capacity of about $180 \mathrm{~g} / \mathrm{g}$ (Fig. $3.3 \mathrm{~b}$ ) The result obtained on the effect of $\mathrm{pH}$ (Fig. $3.3 \mathrm{~b}$ ) on swelling shows that the swelling of the hydrogel increase with initial increase in $\mathrm{pH}$ with a maximum value obtained at $\mathrm{pH} 2$. 

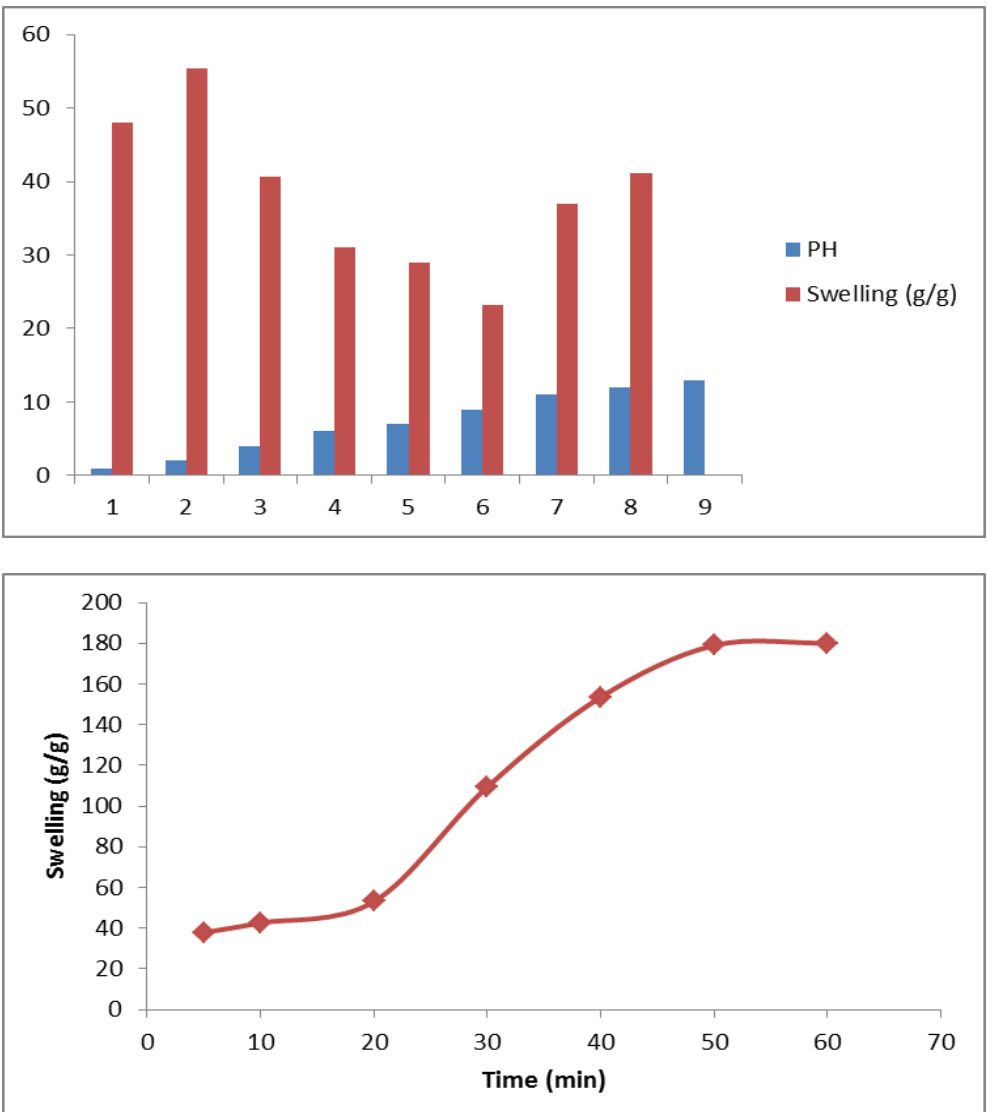

Fig. 3.3: (A \& B): Effect of Contact Time and pH on Swelling Property of Hydrogel.

\subsection{Invitro controlled drug release studies}

The studies on invitro release of fluorouracil as a model drug from the drug loaded biopolymer hydrogel were investigated using process reported elsewhere. Initially, a solution with $\mathrm{pH} 7.4$ was prepared by dissolving the appropriate buffer capsule in $100 \mathrm{~mL}$ capacity volumetric flask. A known quantity of drug loaded hydrogel was immersed into the solution and placed in a dissolution tester under constant stirring for a specified period of time. The time taken was sufficient enough for the release of fluorouracil into the solution. Afterwards, 5 $\mathrm{mL}$ was withdrawn from the test solution and analyzed with UV spectrophotometer (Shimadzu UV/Vis Spectrophotometer UV-1800, Japan). Eventually, a calibration curve was used to determine the amount of drug released. The release process was allowed to run for 5 days following the attainment of constant concentration. The diffusion of the drug from the hydrogel into the release medium is said to occur as a result of penetration of the later into the former. The release medium extracts the drug to form solution. The study revealed that up to $90 \%$ of the drug could be release within 5 days. The initial slow release of drug from the first $24 \mathrm{~h}$ might be attributed to strong affinity of hydrogel for drug on one hand and the degree of cross linking on the other. Finally, the eventual fastness of release occurred due to weakening of cross links in the hydrogel matrix over time.

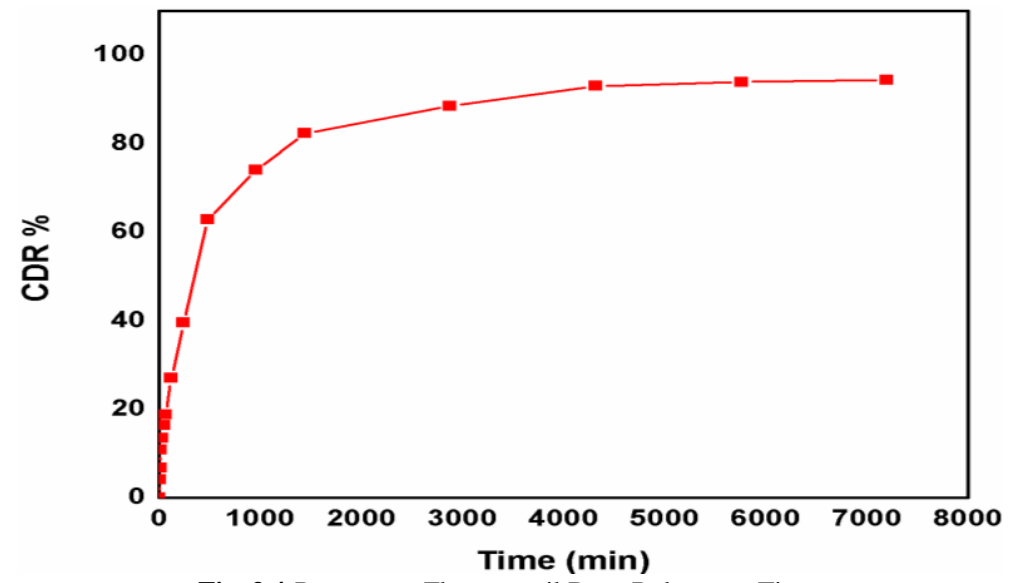

Fig. 3.4:Percentage Fluorouracil Drug Release vs.Time.

\section{Conclusion}

A pullulan derived hydrogel was prepared through free radical graft copolymerization reaction. The hydrogel was evaluated for its structure and morphology using Fourier Transform Infra-red (FT-IR) and Field Emission Scanning Electron Microscopy (FESEM) spectroscopic techniques respectively. The release experiment showed that up to $90 \%$ of modeled drug could be released within 5 days. The prepared hydrogel has proven to be promising for biomedical applications. 


\section{Acknowledgement}

The author gratefully acknowledges the financial support received from the Department of Institution Base Research (IBR) grant, Tertiary Education Trust Fund (TETFUND) through Umaru Ali Shinkafi Polytechnic, Sokoto. Sokoto State, Nigeria.

\section{References}

[1] V. S., Ghorpade et al., "Citric acid crosslinked carboxymethylcellulose-poly(ethylene glycol) hydrogel films for delivery of poorly soluble drugs," International Journal of Biological Macromolecules, pp. 1-28, 2018.https://doi.org/10.1016/j.ijbiomac.2018.06.142.

[2] K. K., Mali, S. C., Dhawale, and R. J. Dias, "Synthesis and characterization of hydrogel films of carboxymethyl tamarind gum using citric acid," International Journal of Biological Macromolecules, vol. 105, pp. 463-470, 2017.https://doi.org/10.1016/j.ijbiomac.2017.07.058.

[3] K., 'Bello, B. K., Sarojini, and B. Narayana, "Design and fabrication of environmentally benign cellulose based hydrogel matrix for selective adsorption of toxic dyes from industrial effluvia," Journal of Polymer Research, vol. 26, p. 62, 2019.https://doi.org/10.1007/s 10965-019-1724-6.

[4] Ferfera-Harrar, N., Aouaz, and N. Dairi, "Environmental-sensitive chitosan-gpolyacrylamide/carboxymethylcellulose superabsorbent composites for wastewater purification I: synthesis and properties," Polym Bull, vol. 73, pp. 815-840, 2016.https://doi.org/10.1007/s00289-015-1521-2

[5] Tally and Y. Atassi, "Synthesis and characterization of pH-sensitive superabsorbent hydrogels based on sodium alginate-g-poly(acrylic acid-coacrylamide) obtained via an anionic surfactant micelle templating under microwave irradiation," Polym Bull, vol. 73, pp. 3183-3208, 2016.https://doi.org/10.1007/s00289-016-1649-8.

[6] H., Mittal, B. S., Kaith, R., Jindal, S. B., Mishra, and A. K. Mishra, "A comparative study on the effect of different reaction conditions on graft copolymerization, swelling, and thermal properties of Gum ghatti-based hydrogels," J Therm Anal Calorim, vol. 119, pp. 131-144, 2015.https://doi.org/10.1007/s10973-014-4140-5.

[7] S., Bashir, Y. Y., Teo, S., Ramesh, and K. Ramesh, "Synthesis and characterization of karaya gum-g- poly (acrylic acid) hydrogels and in vitro release of hydrophobic quercetin," Polymer, p. 071, 2018.https://doi.org/10.1016/j.polymer.2018.05.071.

[8] F., Soleiman, M., Sodaghi, and H. Shahsavari, "Graft copolymerization of gelatin-g-poly (acrylic acid-co-acrylamide) and calculation of grafting parameters," Indian Journal of Science and Technology, pp. 2041-2046, 2012.

[9] P., Wu and M. Imai, "Food polymer pullulan- $\kappa$-carrageenan composite membrane performed smart function both on mass transfer and molecular size recognition," Desalination and Water Treatment, vol. 34, pp. 239-245, 2011.https://doi.org/10.5004/dwt.2011.2872.

[10] H., Li et al., "Superabsorbent polysaccharide hydrogels based on pullulan derivate as antibacterial release wound dressing," JOURNAL OF BIOMEDICAL MATERIALS RESEARCH A, vol. 38A, pp. 31-39, 2011.https://doi.org/10.1002/jbm.a.33045.

[11] X., Tian et al., Carbohydrate Polymers, vol. 87, pp. 1956- 1962, 2012.https://doi.org/10.1016/j.carbpol.2011.10.003

[12] S., Agnihotri and R. Singhal, "Effect of Sodium Alginate Content in Acrylic Acid/Sodium Humate/Sodium Alginate Superabsorbent Hydrogel on Removal Capacity of $\mathrm{MB}$ and CV Dye by Adsorption," Journal of Polymers and the Environment, vol. 27, pp. 372-385, 2019.https://doi.org/10.1007/s10924-018-1349-6.

[13] E., Trovatti et al., "Pullulan-nanofibrillated cellulose composite films with improved thermal and mechanical properties," Composites Science and Technology, vol. 72, pp. 1556-1561, 2012.https://doi.org/10.1016/j.compscitech.2012.06.003. 